\title{
Special section on recommender systems in tourism
}

\author{
Julia Neidhardt $^{1}$ (D) Tsvi Kuflik ${ }^{2} \cdot$ Wolfgang Wörndl $^{3}$
}

Received: 7 April 2018 / Accepted: 11 April 2018 / Published online: 17 April 2018

(C) Springer-Verlag GmbH Germany, part of Springer Nature 2018

The focus of this special section is on the specific challenges for recommender systems in tourism. The common task of classical recommender systems is to find the best (single) product for a user, but in the domain of tourism, the scenario is considerably more complex. Planning a vacation usually involves searching for a reasonably large set of products that are interconnected (e.g., means of transportation, lodging, attractions), with a rather limited availability, and where contextual aspects may have a major impact (e.g., spatiotemporal context, social context, environmental context). In addition and most importantly, products are emotionally "loaded" and therefore decision taking is not based only on rational and objective criteria (Werthner and Ricci 2004). As such, providing the right information to visitors of a tourism site at the right time about the site itself and various services nearby is challenging. Additionally, and in contrast to many other domains, information providers are normally small-medium enterprises (SMEs) that do not have full information about available opportunities. Finally, there is no single, standard format to house this information and with this diversity, building effective recommendation systems within the tourism domain is extremely challenging.

Julia Neidhardt

julia.neidhardt@ec.tuwien.ac.at

Tsvi Kuflik

tsvikak@is.haifa.ac.il

Wolfgang Wörndl

woerndl@in.tum.de

1 Research Division of E-Commerce, TU Wien, Favoritenstrasse 9-11/194-04, 1040 Vienna, Austria

2 Department of Information Systems, University of Haifa, Haifa, Israel

3 Department for Informatics, Technical University of Munich, Munich, Germany 
The rapid development of information and communication technologies (ICT) in general and the web in particular has transformed the tourism domain whereby travelers no longer rely on travel agents/agencies (Werthner et al. 2015). Indeed, recent studies suggest that travelers are now active in searching for information and composing their vacation packages according to their specific preferences (Xiang et al. 2015). When onsite, they search for freely available information about the site itself rather than renting a visitor guide that may be available but considered to be expensive and sometimes outdated. However, like in many other cases, the blessing of the web comes with a curse-the curse of information overload. Recommender systems have been suggested as a practical tool for overcoming this information overload.

The papers in this special section aim to specifically address some of the previously discussed challenges. Two of the papers, i.e., the first and the third one, represent extended versions of contributions presented at RecTour 2016, the Workshop on Recommenders in Tourism that was held in conjunction with the 10th ACM Conference on Recommender Systems (RecSys 2016) in Boston, MA, USA on September 15th, 2016 (Fesenmaier et al. 2016). The second paper was submitted specifically to the special section. While far from being comprehensive, the three selected articles tackle some of the current key challenges that have to be faced in order to build more effective recommendation systems within tourism.

Weather plays a major role in tourism. It must be taken into account while planning a tour as well as during the trip itself as unexpected weather events may impact the original plan. Surprisingly, weather aspects have received little attention in the literature of context-aware mobile recommender systems so far. However, the impact of weather is definitely an area that calls for further research (Gavalas et al. 2014). Hence Trattner et al. investigate in the first paper, which extends their work presented at RecTour 2016 (Trattner et al. 2016), the "Utility of the weather context for point of interest recommendations". Their research contributes to the literature on tourism recommender systems by investigating in detail the extent to which weather features impact user check-in behavior on foursquare and how these features perform in the context of a points of interest (POI) recommender system. The authors suggest a new POI recommendation algorithm and demonstrate that the weather context can significantly increase the recommender accuracy of a POI recommendation.

The title of the second paper is "Estimating a latent-class user model for travel recommender systems". When selecting the sites to visit on a trip, tourists have to trade-off attraction against routing and time-use characteristics of POIs. In order to recommend optimal personalized travel plans, an accurate assessment of how users make these trade-offs is important. In this paper, Arentze et al. report the results of a study conducted to estimate a user model for travel recommender systems that takes into account a multi-attribute utility function of POIs as well as dynamic needs of persons on a trip. A latent-class analysis of the results of an online survey shows that significant differences exist between tourists in terms of how they make the tradeoffs between the factors and respond to needs. The estimation results provide the parameters of a multi-class user model that can be used within travel recommender systems. 
Finally, the third paper takes a look at group recommendation in tourism. Recommender systems are usually designed to suggest items to individual users. However, as many activities are typically done within group, group recommender systems have become an important focus of research. Particularly in the travel domain it is crucial to target families or group of friends, as traveling is often a social activity. However, in order to develop travel recommender systems that are truly beneficial for groups, the dynamics of group decision making processes in the tourism domain have to be better understood. Delic et al. address this problem in the paper "An observational user study for group recommender systems in the tourism domain", which extends their work presented at RecTour 2016 (Delic et al. 2016). They illustrate the design, the outcome and the implications of a study that observes groups before, during and after a travel decision making process.

The three papers included in this special section shed light on several specific and less explored aspects of the challenges in the complex domain of recommender systems for tourism.

\section{References}

Delic A, Neidhardt J, Nguyen TN, Ricci F (2016) Research methods for group recommender systems. In: Proceedings of RecTour 2016, 15th Sep 2016, Boston, CEUR-WS.org/Vol-1685

Fesenmaier DR, Kuflik T, Neidhardt J (2016) RecTour 2016: workshop on recommenders in tourism. In: Proceedings of the 10th ACM conference on recommender systems. ACM, New York, pp 417-418

Gavalas D, Konstantopoulos C, Mastakas K, Pantziou G (2014) Mobile recommender systems in tourism. J Netw Comput Appl 39:319-333

Trattner C, Oberegger A, Eberhard L, Parra D, Marinho LB (2016) Understanding the impact of weather for POI recommendations. In: Proceedings of RecTour 2016, 15th Sep 2016, Boston, CEUR-WS. org/Vol-1685

Werthner H, Ricci F (2004) E-commerce and tourism. Commun ACM 47(12):101-105

Werthner H, Alzua-Sorzabal A, Cantoni L, Dickinger A, Gretzel U, Jannach D, Neidhardt J, Pröll B, Ricci F, Scaglione M, Stangl B, Stock O, Zanker M (2015) Future research issues in IT and tourism. J IT Tour 15(1):1-15

Xiang Z, Magnini VP, Fesenmaier DR (2015) Information technology and consumer behavior in travel and tourism: insights from travel planning using the internet. J Retail Consum Serv 22:244-249 